
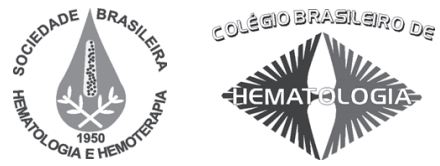

\title{
Expansão de células-tronco da medula óssea e do sangue de cordão umbilical humano
}

\author{
Expansion of stem cells from bone marrow and human umbilical cord blood
}

Alexandra C. Senegaglia ${ }^{1}$

Carmen L. K. Rebelatto ${ }^{I}$

Paula H. Suss ${ }^{l}$

Paulo R. S. Brofman ${ }^{2}$
Células-tronco/progenitoras frequentemente não estão disponíveis em quantidade suficiente para restauração de órgãos e tecidos danificados, sendo necessária sua expansão in vitro. Instalações físicas adequadas, pessoal técnico qualificado, reagentes de grau clínico e protocolos bem definidos de acordo com as condições de boas práticas de fabricação são imprescindíveis para assegurar a qualidade e segurança das células infundidas no paciente. A medula óssea e o sangue de cordão umbilical ainda são as fontes de células mais utilizadas em terapias. Protocolos bem sucedidos de expansão utilizando células-tronco hematopoéticas, células-tronco mesenquimais e células progenitoras endoteliais já têm sido empregados em estudos pré-clínicos e clínicos. A escolha do tipo celular adequado deve ser direcionada pelo tamanho da lesão ou natureza do tecido tratado e pelo efeito terapêutico desejado. Estudos recentes têm demonstrado que propriedades de diferentes células expandidas in vitro podem ser combinadas para obtenção de um resultado melhor no tratamento de algumas doenças. Células em culturas de longo termo precisam ser acompanhadas por meio de diversas técnicas de citogenética clássica e molecular para demonstrar que não há evidências de transformação espontânea ou sinais de imortalização. Ensaios utilizando a infusão de células expandidas através da barreira alogeneica e xenogeneica, apresentaram melhora funcional e foram alcançados sem imunossupressão e sem evidencias de infiltrados celulares que indicariam resposta imune. Porém, mais estudos precisam ser realizados para avaliar a imunogenicidade destas células e garantir a segurança da terapia celular alogênica permitindo sua consolidação no uso clínico. Aqui apresentamos uma atualização sobre expansão celular associada com seu uso clínico. Rev. Bras. Hematol. Hemoter. 2009;31(Supl. 1):9-14.

Palavras-chave: Células-tronco; medula óssea; cordão umbilical.

\section{Introdução}

As células-tronco/progenitoras não estão disponíveis, naturalmente, em quantidade suficiente para restauração de órgãos e tecidos danificados. Para assegurar o sucesso das terapias regenerativas é necessária a expansão do número destas células. Algumas das limitações ao desenvolvimento de terapias utilizando células expandidas in vitro são as necessidades de instalações físicas adequadas, pessoal técnico qualificado com experiência em cultivo e protocolos bem definidos para a produção de células, seguindo todas as normas internacionais de boas práticas de fabricação. Outra dificuldade é que a expansão requer uma grande variedade de reagentes, como citocinas e fatores de crescimento, que, em sua grande maioria, não são produzidos em grau clínico. A necessidade da substituição destes reagentes por similares

${ }^{1}$ Bióloga - Pontifícia Universidade Católica do Paraná (PUCPR) - Curitiba-PR.

${ }^{2}$ Professor Titular - Pontificia Universidade Católica do Paraná (PUCPR) - Curitiba-PR

Núcleo de Cardiomioplastia Celular - Pontifícia Universidade Católica do Paraná - Curitiba-PR.

Correspondência: Alexandra Cristina Senegaglia

Rua Guilherme Pugsley, 1760 ap 501

80620-000 - Curitiba-PR - Brasil

Tel.: (55 41) 3271-2219 - Fax.: (55 41) 3271-1621

E-mail: alexandra.senegaglia@pucpr.br

Doi: 10.1590/S1516-84842009005000020 
de grau clínico é crítica para o desenvolvimento de novos protocolos. Este procedimento visa não somente minimizar os riscos de contaminação, mas também reduzir a chance de desvios de protocolos e otimizar a identificação de problemas na produção e melhorar o processo como um todo. ${ }^{1}$

A escolha do tipo celular adequado para a terapia celular deve ser direcionada pelo tamanho da lesão ou natureza do tecido que está sendo tratado e pelo efeito terapêutico desejado. A expansão de células específicas visa a melhora da eficácia da terapia celular, diminuindo a possibilidade de expansão inesperada, in vivo, de células não adequadas. As principais fontes de células utilizadas atualmente são a medula óssea e o sangue de cordão umbilical humano (SCUH).

\section{Medula óssea}

O transplante de medula óssea, utilizado mais comumente para recuperar a linhagem hematopoética, foi o primeiro tipo de transplante que abordou a terapia com células-tronco, e os primeiros ensaios clínicos com sucesso foram na década de $1950 .^{2}$

O uso da medula óssea como fonte de células, especialmente para terapia em cardiomiopatias, tem alguns pontos desfavoráveis, tais como o risco em relação ao procedimento da coleta, a possibilidade de formação de trombos se induzida à mobilização no sangue periférico e a dificuldade no uso para o tratamento da doença isquêmica aguda. ${ }^{3} \mathrm{~A}$ atividade das células-tronco e especialmente das células progenitoras endoteliais é diminuída e o seu número é reduzido em pacientes que sofrem de doenças cardiovasculares, bem como nos pacientes de idade avançada. ${ }^{4,5,6}$

Entre os pontos favoráveis ao uso da medula óssea, está a possibilidade do transplante autólogo e de não haver necessidade de criopreservação da amostra para uso posterior, podendo-se obter uma grande quantidade de células que, no caso dos transplantes com células-tronco hematopoéticas, em geral, é suficiente para o sucesso do transplante.

\section{Sangue de cordão umbilical humano}

O primeiro transplante utilizando células do sangue de cordão umbilical humano (SCUH) foi realizado em 1988 por Gluckman e colaboradores ${ }^{7}$ para tratar uma criança com anemia de Fanconi. Até o momento, mais de 10 mil transplantes com SCUH já foram realizados em todo o mundo. Atualmente existem mais de duzentos bancos públicos e privados de SCUH no mundo, armazenando em torno de 2 milhões de unidades. ${ }^{8}$

Em relação ao SCUH têm-se como desvantagens a necessidade da criopreservação para um possível uso futuro e o número de células limitado ao volume coletado. Dentre as vantagens, a coleta não envolve nenhum tipo de risco para a mãe ou para o bebê, e as células podem ser facilmente extraídas e criopreservadas. Em comparação com as células da medula óssea, no SCUH existe frequentemente um número maior de células-tronco por volume coletado, com alta capacidade de proliferação, ${ }^{3}$ e as células transplantadas são menos suscetíveis ao desencadeamento da reatividade alogênica no receptor. ${ }^{9}$

\section{Células-tronco mesenquimais}

As células-tronco mesenquimais (CTM) contribuem na regeneração de cartilagem, gordura, ossos, músculos e estroma, ${ }^{10}$ e a expansão destas células, provenientes de diversas fontes, tem sido explorada para o uso clínico nas mais variadas áreas. ${ }^{11,12,13}$ Porém, não demonstram in vivo capacidade de regeneração ou manutenção de um compartimento tecidual. ${ }^{14} \mathrm{CTM}$ proliferam em cultura aderidas ao plástico, possuem capacidade de autorrenovação e podem diferenciar-se in vitro em múltiplas linhagens; representam uma fração muito pequena, apenas $0,001 \%$ a $0,01 \%$ das células nucleadas da medula óssea ${ }^{10}$ e $0,00003 \%$ das células nucleadas do SCUH. ${ }^{15}$ A caracterização celular destas células ainda não está totalmente definida, mas há um consenso de que devam ser positivas para os marcadores CD29, CD44, CD90, CD105, CD73 e negativas para os marcadores CD34, CD45, $\mathrm{CD} 14$ e CD3., ${ }^{1,16,17}$

A utilização das CTM no transplante para o tratamento de doenças hematopoéticas malignas e não malignas é fundamentada na sua aparente habilidade de modulação imunológica.

A doença do enxerto contra o hospedeiro (DECH) é uma complicação pós-transplante de células-tronco hematopoéticas (CTH) que apresenta grande índice de mortalidade. As CTM dão suporte para o crescimento e diferenciação de CTH no microambiente medular e promovem a pega do enxerto em modelos animais. ${ }^{18}$ Em um estudo com 55 pacientes, resistentes ao tratamento com esteróides e com DECH de graus II a IV, realizado por Le Blanc e colaboradores, ${ }^{19}$ as CTM dos doadores, expandidas in vitro, foram infundidas nos pacientes pós-transplante de CTH. Após 60 meses de acompanhamento, 39 pacientes responderam ao tratamento, com sobrevida significativamente mais alta naqueles com resposta completa e sem efeitos colaterais, sugerindo que as CTM podem suprimir a resposta das células $\mathrm{T}$ do doador aos aloantígenos do receptor.

Ball e colaboradores, ${ }^{20} \mathrm{em}$ um ensaio clínico envolvendo 14 pacientes, cotransplantaram CTM e CTH haploidênticas. Cinco semanas antes do transplante de $\mathrm{CTH}$, células mononucleares foram isoladas da medula óssea e as CTM foram expandidas in vitro. Comparando-se os resultados dos pacientes que receberam a coinfusão de células com um histórico de 47 pacientes (do grupo controle) nas mesmas condições, que não receberam o cotransplante, verificou-se que no grupo controle houve $15 \%$ de falha na pega do transplan- 
te e no grupo que recebeu as CTM todos os pacientes tiveram pega do enxerto sem nenhuma reação adversa, demonstrando que as CTM possivelmente suprimem o efeito alorreativo dos linfócitos $\mathrm{T}$ do hospedeiro.

A expansão in vitro possui o risco de acumular mudanças genéticas e epigenéticas na célula, o que poderia levar a uma transformação celular e ao câncer. ${ }^{21} \mathrm{O}$ cultivo de CTM na presença de reagentes de origem xenogeneica também limita a utilização destas células em ensaios clínicos.

O uso do soro autólogo do paciente em substituição ao soro bovino fetal tem como fator limitante a quantidade que pode ser obtida e também o fato de que a idade dos indivíduos dos quais o soro foi obtido interfere na proliferação e/ou na diferenciação das CTM. ${ }^{22}$ Schallmoser e colaboradores $^{23}$ demonstraram a capacidade de substituir o uso de soro bovino fetal pelo lisado de plaquetas no cultivo de CTM. Este procedimento previne a contaminação das células cultivadas com patógenos bovinos e a xenoimunização, mantendo a capacidade de proliferação e diferenciação, e assim permite a utilização clínica das células cultivadas em diferentes doenças. O lisado de plaquetas é obtido a partir da concentração de dez unidades de plaquetas humanas ricas em plasma. Cada unidade é constituída de uma unidade de plasma de doador do grupo $\mathrm{AB}$ e quatro unidades de concentrado de plaquetas, de doadores do grupo $\mathrm{O}$, previamente filtradas para depleção de células brancas. A lise das plaquetas se dá pelo congelamento a $-30^{\circ} \mathrm{C}$; a seguir o produto é centrifugado e filtrado, resultando em um volume aproximado de 2,5 a 3,0 litros, o qual é aliquotado e congelado para uso posterior.

Em culturas de longo termo, CTM de camundongo acumularam aberrações cromossômicas e exibiram um fenótipo de transformação maligno ${ }^{24} \mathrm{e}$, em alguns modelos animais, estas células levaram à formação de tumores in vivo. ${ }^{25,26} \mathrm{CTM}$ derivadas do tecido adiposo demonstraram potencial de transformação espontânea após culturas de longo termo in vitro. ${ }^{27}$ CTM humanas originadas da medula óssea parecem diferir bastante no comportamento quando comparadas às CTM de camundongo. Bernardo e colaboradore ${ }^{28}$ realizaram um estudo utilizando diversas técnicas de citogenética clássica e molecular para demonstrar que não há evidência de transformação espontânea ou sinal de imortalização das CTM obtidas da medula óssea de indivíduos saudáveis em culturas de longo termo. A análise em diferentes pontos do cultivo que alcançou 25 passagens, ou até a senescência das células, mostrou que não houve aparecimento de anormalidades cromossômicas, a atividade da telomerase e dos transcritos do gene hTERT não foram expressos e os telômeros sofreram encurtamento durante o período de cultivo.

\section{Hemoangioblastos}

O estabelecimento de ilhas sanguíneas no saco vitelínico marca o início da hematopoese e da vasculogênese no desenvolvimento embrionário de camundongos; essas deri- vam de agregados de células mesodermais e colonizam o saco vitelínico pós-fecundação. As células centrais no interior desses agregados originam as células hematopoéticas embrionárias, enquanto a população periférica se diferencia nas células endoteliais que formam as primeiras estruturas vasculares. ${ }^{29,30,31}$ Esse desenvolvimento próximo entre a linhagem hematopoética que origina as células do sistema sanguíneo e da linhagem endotelial leva à hipótese de que elas derivam de um precursor ancestral comum, o hemo-

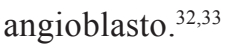

Em estudos in vitro identificou-se uma população de precursores embrionários com potencial para originar as linhagens hematopoética e endotelial. Esses precursores desenvolvem-se em uma mesma célula, denominada célula formadora de colônias de blastos (BL-CFC), a qual está presente logo no início do desenvolvimento do corpo embrióide e persiste por um curto período precedendo o início da hematopoese e da vasculogênese. ${ }^{31}$ As células CD133+ são originadas a partir da BL-CFC e preenchem muitos critérios, os quais caracterizam os verdadeiros hemoangioblastos.

\section{Células-tronco hematopoéticas}

A expansão de CTH in vitro envolve o cultivo das células em suspensão na presença de meio e várias combinações de citocinas e fatores de crescimento. $\mathrm{O}$ aumento no número de CTH é especialmente útil para um melhor aproveitamento das amostras de SCUH criopreservadas. O baixo número de $\mathrm{CTH}$ obtidas se deve ao pequeno volume de sangue coletado, o que limita sua utilização para crianças ou adultos de baixo peso. A expansão ex vivo de CTH do SCUH tornaria esta importante fonte de células útil para aplicações também em adultos. Por isso, nas últimas duas décadas, numerosas tentativas têm sido realizadas para expandir CTH em cultura. ${ }^{34-38}$

Uma alternativa tem sido a realização da expansão $e x$ vivo utilizando-se como substrato nanofibras com grupamentos amina conjugados à superfície..$^{39}$ As nanofibras parecem prover superfícies adesivas que promovem a expansão das CTH na presença do SCF, Flt-3 ligante, TPO e IL-3. Ao final de dez dias, os espaçadores de etileno e butilieno mostraram alta eficiência de expansão, aumentaram em 200 e 235 vezes, respectivamente, o número de células CD34+, CD45+ com $95 \%$ de viabilidade e foram capazes de repovoar a medula de camundongos diabéticos não obesos e com imunodeficiência severa combinada NOD/SCID.

Outro estudo recente ${ }^{40}$ demonstrou que um grupo de proteínas semelhantes à angiopoietina, especialmente a 5 (Angptls-5), e o fator de crescimento de insulina ligante da proteína 2 (IGFBP2), quando associados a outros fatores, podem expandir CTH derivadas do $\mathrm{SCUH}$ em até vinte vezes e também repovoar a medula de camundongos NOD/SCID.

A expansão in vitro de células da linhagem eritroide pode ser uma alternativa devido à diminuição de doadores 
de sangue ou no caso de pacientes de grupos sanguíneos raros. Vários ensaios têm sido realizados na intenção de produzir células vermelhas in vitro. ${ }^{41,43}$ Baek e colaboradores $^{43}$ descreveram a geração in vitro de células eritroides derivadas de células CD34+ de SCUH. As células mononucleares foram selecionadas positivamente para o CD34+ e foram cultivadas, por uma semana, em meio livre de soro, suplementado, acrescido de fator de crescimento de célulastronco (SCF), interleucina 3 (IL-3) e eritropoietina (EPO) ou SCF, IL-3 e trombopoietina (TPO) mais o Flt-3 e posteriormente cocultivadas com CTM derivadas do SCUH e da medula óssea. Os melhores resultados foram obtidos no meio com EPO e no cocultivo com CTM do SCUH. As células $\mathrm{CD} 34+$ foram diferenciadas na linhagem eritroide e puderam ser expandidas em larga escala, fornecendo uma base sólida para futuras produções clínicas de células vermelhas para transfusão.

\section{Células progenitoras endoteliais}

Até recentemente acreditava-se que a angiogênese, que é a formação de novos vasos sanguíneos, a partir daqueles pré-existentes ${ }^{44,45}$ era a única forma de indução da neovascularização em tecidos isquêmicos. Evidências sugerem que a vasculogênese, que implica a diferenciação in situ dos hemoangioblastos em células endoteliais e sua subsequente organização em redes capilares primárias, não ocorre apenas no estágio embrionário. O estudo de Asahara e colaboradores,$^{30} \mathrm{em} \mathrm{1997}$, foi fundamental para demonstrar a vasculogênese pós-natal e a existência das células progenitoras endoteliais (CPE).

Estratégias terapêuticas promissoras são baseadas no conceito de que as CPE podem diferenciar-se em células semelhantes às endoteliais, contribuindo para os processos de reparo vascular. ${ }^{46}$ Aproximadamente $0,01 \%$ das células mononucleares da medula óssea são $\mathrm{CPE},{ }^{47}$ o número de $\mathrm{CPE}$ no sangue periférico é 500 vezes menor que na medula ós$\mathrm{sea}^{48} \mathrm{e}$ no SCUH foram encontradas $0,64 \%$ de CPE. ${ }^{49}$

Em estudo pré-clínico de Kawamoto e colaboradores, ${ }^{50}$ as CPE diferenciadas in vitro e infundidas na área isquêmica de ratos com enfarte agudo do miocárdio foram detectadas na área de neovascularização em estudos histológicos pós-eutanásia. Demonstrou-se que essas células não participam apenas da indução de fatores parácrinos, os quais estimulariam a proliferação de células presentes in situ, mas sim fisicamente da reconstituição da área lesada. Estudo similar foi realizado por Kalka e colaboradores ${ }^{51}$ em um modelo de ratos com isquemia de membro inferior, também apresentando melhora nos animais que receberam as CPE expandidas.

Até o presente, o transplante de CPE derivadas do SCUH ainda encontra-se na fase de experimentos pré-clíni$\cos .^{3} \mathrm{~A}$ exata população de CPE (purificadas ou diferenciadas) que seria mais adequada para o uso na terapia celular ainda não está definida. Em modelos animais, ambos os tipos têm se mostrado efetivos na melhora da neovascularização, como demostrado pelos experimentos de Melero-Martin e colaboradores. ${ }^{52}$

Estudos recentes têm demonstrado que as propriedades diferentes das CTM e das CPE podem ser combinadas para obtenção de um resultado melhor no tratamento de cardiomiopatias. Ensaios pré-clínicos em ratos utilizando a coinfusão das CTM e das CPE mostraram um aumento na expressão de fatores angiogênicos e menor deposição de colágeno, poucas células em apoptose e uma melhora regional do fluxo sanguíneo quando comparados ao grupo controle, sugerindo que o transplante combinado destas células deve ser considerado para melhora da função cardíaca. ${ }^{53}$

\section{Transplantes alogênicos}

Têm sido relatados estudos utilizando a infusão de CTM através da barreira alogeneica e xenogeneica, apresentando algum tipo de recuperação. Estes resultados foram alcançados sem imunossupressão e sem evidências de infiltrados celulares que indicariam resposta imune..$^{54-56}$

Cho e colaboradores ${ }^{57}$ demonstraram em um experimento com miniporcos, com o complexo principal de histocompatibilidade (CPH) definido, a imunogenicidade das CTM de SCUH. A conclusão do estudo é que uma única infusão de células não compatíveis para o complexo principal de histocompatibilidade não induz a uma resposta imune detectável. No entanto, estas células são imunogênicas quando a infusão é realizada em uma região de inflamação, ou são realizadas infusões repetidas em uma mesma região, ou ainda quando estimuladas com o interferon gama (IFN- $\gamma$ ); antes da infusão estas células são imunogênicas. Como algumas vezes estratégias de terapia celular envolvem a infusão de células em regiões de inflamação, ou repetidas doses, estes resultados podem ter implicações importantes para se alcançarem bons resultados sem efeitos deletérios. Estudos mais aprofundados com as CTM e outros tipos de células expandidas em cultivo precisam ser realizados com o objetivo de garantir a segurança da terapia celular alogênica para que futuramente seu uso clínico seja consolidado.

\footnotetext{
Abstract

Stem/progenitor cells are not frequently available in large enough amounts to repair damaged tissues and organs and so in vitro expansion is necessary. Appropriate facilities, qualified technicians, clinical-grade reagents and well defined protocols relating to good manufacturing products are essential to assure the quality and security of the cells injected in the patient. Bone marrow and human umbilical cord blood are still the best sources of cells for therapies. Successful expansion protocols using hematopoietic stem cells, mesenchymal stem cells and endothelial progenitor cells have already been used in clinical and pre-clinical trials. Adequate cell choice should consider the extent of injury or nature of the damaged
} 
tissue and the desired therapeutic effect. Recent studies have demonstrated that properties of different in vitro expanded cells can be combined aiming to improve the outcome of the treatment of some diseases. Long-term cell cultures need to be followed up by classical and molecular cytogenetic techniques to demonstrate that there is no evidence of spontaneous transformation or signs of immortalization. Assays using expanded cell infusions across both xenogeneic and allogeneic transplant barriers showed functional improvement and were achieved without immunosuppression and without evidence of a cellular infiltrate that would indicate an immune response. However, more research needs to be performed to evaluate the immunogenicity of these cells and to guarantee the safety of allogeneic cell therapy, allowing consolidation of their clinical use. Here, we present an update regarding cellular expansion associated with their clinical use. Rev. Bras. Hematol. Hemoter. 2009;31(Supl. 1):9-14.

Key words: Stem cell; bone marrow; cord blood.

\section{Referências Bibliográficas}

1. Wagner JE, Verfaillie CM. Ex vivo expansion of umbilical cord blood hemopoietic stem and progenitor cells. Exp Hematol. 2004; $32(5): 412-3$.

2. Thomas ED, Lochte HL Jr, Lu WC, Ferrebee Jw. Intravenous infusion of bone marrow in patients receiving radiation and chemotherapy. N Engl J Med. 1957;257(11):491-6.

3. Zhang L, Yang R, Han ZC. Transplantation of umbilical cord blood-derived endothelial progenitor cells: a promising method of therapeutic revascularization. Eur J Haematol. 2006; 76 (1): $1-8$.

4. Couffinhal T, Silver M, Kearney M, Sullivan A, Witzenbichler B, Magner M, et al. Impaired collateral vessel development associated with reduced expression of vascular endothelial growth factor in ApoE-/-mice. Circulation. 1999;99(24):3188-98.

5. Van Belle E, Rivard A, Chen D, Silver M, Bunting S, Ferrara N, et al. Hypercholesterolemia attenuates angiogenesis but does not preclude augmentation by angiogenic cytokines. Circulation. 1997;96(8):2667-74.

6. Rivard A, Fabre JE, Silver M, Chen D, Murohara T, Kearney M, et al. Age-dependent impairment of angiogenesis. Circulation. 1999; 99(1):111-20.

7. Gluckman E, Broxmeyer HA, Auerbach AD, Friedman HS, Douglas GW, Devergie A, et al. Hematopoietic reconstitution in a patient with Fanconi's anemia by means of umbilical-cord blood from an HLA-identical sibling. N Engl J Med. 1989;321(17):1174-8.

8. Verter. http://parentsguidecordblood.org

9. Rocha V, Wagner JE Jr, Sobocinski KA, Klein JP, Zhang MJ, Horowitz MM, et al. Graft-versus-host disease in children who have received a cord-blood or bone marrow transplant from an HLA-identical sibling. Eurocord and International Bone Marrow Transplant Registry Working Committee on Alternative Donor and Stem Cell Sources. N Engl J Med. 2000;342(25):1846-54.

10. Pittenger MF, Mackay AM, Beck SC, Jaiswal RK, Douglas R, Mosca JD, et al. Multilineage potential of adult human mesenchymal stem cells. 1999;284(5411):143-7.

11. Horwitz EM, Gordon PL, Koo WK, Marx JC, Neel MD, McNall $\mathrm{RY}$, et al. Isolated allogeneic bone marrow-derived mesenchymal cells engraft and stimulate growth in children with osteogenesis imperfecta: Implications for cell therapy of bone. Proc Natl Acad Sci USA. 2002;99(13):8932-7.
12. Le Blanc K. Mesenchymal stromal cells: Tissue repair and immune modulation. Cytotherapy. 2006;8(6):559-61

13. Makino S, Fukuda K, Miyoshi S, Konishi F, Kodama H, Pan J, et al. Cardiomyocytes can be generated from marrow stromal cells in vitro. J Clin Invest. 1999;103(5):697-705.

14. Javazon EH, Beggs KJ, Flake AW. Mesenchymal stem cells: paradoxes of passaging. Exp Hematol. 2004;32(5):414-25.

15. Campagnoli C, Roberts IA, Kumar S, Bennett PR, Bellantuono I, Fisk NM. Identification of mesenchymal stem/progenitor cells in human first-trimester fetal blood, liver, and bone marrow. Blood. 2001;98(8):2396-402.

16. Kern S, Eichler H, Stoeve J, Klüter H, Bieback K. Comparative analysis of mesenchymal stem cells from bone marrow, umbilical cord blood, or adipose tissue. Stem Cells. 2006;24(5):1294-301.

17 Rebelatto CK, Aguiar AM, Moretão MP, Senegaglia AC, Hansen P, Barchiki $\mathrm{F}$, et al. Dissimilar differentiation of mesenchymal stem cells from bone marrow, umbilical cord blood, and adipose tissue. Exp Biol Med (Maywood). 2008;233(7):901-13.

18. Noort WA, Kruisselbrink AB, in't Anker PS, Kruger M, van Bezooijen RL, de Paus RA, et al. Mesenchymal stem cells promote engraftment of human umbilical cord blood-derived CD34(+) cells in NOD/SCID mice. Exp Hematol. 2002;30(8):870-8.

19. Le Blanc K, Frassoni F, Ball L, Locatelli F, Roelofs H, Lewis I, et al. Mesenchymal stem cells for treatment of steroid-resistant, severe, acute graft-versus-host disease: a phase II study. Lancet. 2008;371(9624):1579-86.

20. Ball LM, Bernardo ME, Roelofs H, Lankester A, Cometa A, Egeler RM, et al. Cotransplantation of ex vivo expanded mesenchymal stem cells accelerates lymphocyte recovery and may reduce the risk of graft failure in haploidentical hematopoietic stem-cell transplantation. Blood. 2007;110(7):2764-7.

21. Lepperdinger G, Brunauer R, Jamnig A, et al. Controversial Issue: Is it safe employ mesenchymal stem cells in cell-based therapies? Exp. Geront. 2008; doi:10.1016/j.exger.2008.07.004 on line published.

22. Mannello F, Tonti GA. Concise review: no breakthroughs for human mesenchymal and embryonic stem cell culture: conditioned medium, feeder layer, or feeder-free; medium with fetal calf serum, human serum, or enriched plasma; serum-free, serum replacement nonconditioned medium, or ad hoc formula? All that glitters is not gold! All that glitters is not gold! Stem Cells. 2007; 25 (7):1 603-9.

23. Schallmoser K, Bartmann C, Rohde E, Reinisch A, Kashofer K, Stadelmeyer E, et al. Human platelet lysate can replace fetal bovine serum for clinical-scale expansion of functional mesenchymal stromal cells. Transfusion. 2007;47(8):1436-46.

24. Zhou YF, Bosch-Marce M, Okuyama H, Krishnamachary B, Kimura $\mathrm{H}$, Zhang L, et al. Spontaneous transformation of cultured mouse bone marrow-derived stromal cells. Cancer Res. 2006; 66(22): 10849-54

25. Miura M, Miura Y, Padilla-Nash HM, Molinolo AA, Fu B, Patel V, et al. Accumulated chromosomal instability in murine bone marrow mesenchymal stem cells leads to malignant transformation. Stem Cells. 2006;24(4):1095-103

26. Aguilar S, Nye E, Chan J, Loebinger M, Spencer-Dene B, Fisk N, et al. Murine but not human mesenchymal stem cells generate osteosarcomalike lesions in the lung. Stem Cells. 2007;25(6):1586-94.

27. Rubio D, Garcia-Castro J, Martín MC, de la Fuente R, Cigudosa JC, Lloyd AC, et al. Spontaneous human adult stem cell transformation. Cancer Res. 2005;65(8):3035-9.

28. Bernardo ME, Zaffaroni N, Novara F, Cometa AM, Avanzini MA, Moretta A, et al. Human bone marrow derived mesenchymal stem 
cells do not undergo transformation after long-term in vitro culture and do not exhibit telomere maintenance mechanisms. Cancer Res. 2007;67(19):9142-9.

29. Asahara T, Bauters C, Zheng LP, Takeshita S, Bunting S, Ferrara N, et al. Synergistic effect of vascular endothelial growth factor and basic fibroblast growth factor on angiogenesis in vivo. Circulation. 1995;92(9 Supp1):II365-71.

30. Asahara T, Murohara T, Sullivan A, Silver M, van der Zee R, Li T, et al. Isolation of putative progenitor endothelial cells for angiogenesis. Science. 1997;275(5302):964-7.

31. Choi K, Kennedy M, Kazarov A, Papadimitriou JC, Keller G. A common precursor for hematopoietic and endothelial cells. Development. 1998;125(4):725-32.

32. Risau W, Flamme I. Vasculogenesis. Annu Rev Cell Dev Biol. 1995;11:73-91.

33. Sabin FR. Preliminary note on the differentiation of angioblasts and the method by which they produce blood-vessels, blood-plasma and red blood-cells as seen in the living chick. Anat. Rec.1917; 13(4):199-204

34. Gammaitoni L, Weisel KC, Gunetti M, Wu KD, Bruno S, Pinelli S, et al. Elevated telomerase activity and minimal telomere loss in cord blood long-term cultures with extensive stem cell replication. Blood. 2004;103(12):4440-8.

35. Summers YJ, Heyworth CM, de Wynter EA, Hart CA, Chang J. Testa NG. AC133+ G0 cells from cord blood show a high incidence of long-term culture-initiating cells and a capacity for more than 100 million-fold amplification of colony-forming cells in vitro. Stem Cells. 2004;22(5):704-15.

36. Araki H, Mahmud N, Milhem M, Nunez R, Xu M, Beam CA, Hoffman R. Expansion of human umbilical cord blood SCIDrepopulating cells using chromatin-modifying agents. Exp Hematol. 2006;34(2):140-9.

37. Suzuki T, Yokoyama Y, Kumano K, Takanashi M, Kozuma S, Takato $\mathrm{T}$, et al. Highly efficient ex vivo expansion of human hematopoietic stem cells using Delta1-Fc chimeric protein. Stem Cells. 2006;24(11):2456-65

38. Amsellem S, Pflumio F, Bardinet D, Izac B, Charneau P, Romeo $\mathrm{PH}$, et al. Ex vivo expansion of human hematopoietic stem cells by direct delivery of the HOXB4 homeoprotein. Nat Med. 2003; 9(11):1423-7.

39. Chua KN, Chai C, Lee PC, Ramakrishna S, Leong KW, Mao HQ. Functional nanofiber scaffolds with different spacers modulate adhesion and expansion of cryopreserved umbilical cord blood hematopoietic stem/progenitor cells. Exp Hematol. 2007;35(5): 771-81.

40. Zhang CC, Kaba M, Iizuka S, Huynh H, Lodish HF. Angiopoietinlike 5 and IGFBP2 stimulate ex vivo expansion of human cord blood hematopoietic stem cells as assayed by NOD/SCID transplantation. Blood. 2008;111(7):3415-23

41. Giarratana MC, Kobari L, Lapillonne H, Chalmers D, Kiger L, Cynober T, et al. Ex vivo generation of fully mature human red blood cells from hematopoietic stem cells. Nat Biotechnol. 2005;23(1):69-74.

42. Loeuillet C, Bernard G, Rémy-Martin J, Saas P, Hervé P, Douay L, et al. Distinct hematopoietic support by two human stromal cell lines. Exp Hematol. 2001;29(6):736-45.

43. Baek EJ, Kim HS, Kim S, et al. In vitro clinical-grade generation of red blood cells from human umbilical cord blood CD34+ cells. Transfusion. 2008; publicado on line 31 de julho.

44. Asahara T, Masuda H, Takahashi T, Kalka C, Pastore C, Silver M, et al. Bone marrow origin of endothelial progenitor cells responsible for postnatal vasculogenesis in physiological and pathological neovascularization. Circ Res. 1999;85(3):221-8.
45. Chachques JC, Duarte F, Herreros J, Prosper F, Giambroni R, Julia P, et al. Cellular myogenic and angiogenic therapy for patients with cardiac or limb ischemia. Basic. Appl. Myol. 2003; 13(1): 29-37.

46. Eggermann J, Kliche S, Jarmy G, Hoffmann K, Mayr-Beyrle U, Debatin KM, et al. Endothelial progenitor cell culture and differentiation in vitro: a methodological comparison using human umbilical cord blood. Cardiovasc Res. 2003;58(2):478-86.

47. Gross P, Herbrig K. Role of endothelial progenitor cells in cardiovascular pathology. Rocz Akad Med Bialymst. 2004;49:174-7.

48. Tateishi-Yuyama E, Matsubara H, Murohara T, Ikeda U, Shintani $\mathrm{S}$, Masaki $\mathrm{H}$, et al. Therapeutic angiogenesis for patients with limb ischaemia by autologous transplantation of bone-marrow cells: a pilot study and a randomised controlled trial. Lancet. 2002;360 (9331):427-35.

49. Senegaglia AC, Brofman PRS, Aita CAM. Células progenitoras endoteliais de sangue de cordão umbilical humano: purificação, expansão e diferenciação [Tese de doutorado]. Curitiba: Pontifícia Universidade Católica do Paraná, 2007. 125p.

50. Kawamoto A, Gwon HC, Iwaguro H, Yamaguchi JI, Uchida S, Masuda $\mathrm{H}$, et al. Therapeutic potential of ex vivo expanded endothelial progenitor cells for myocardial ischemia. Circulation. 2001;103(5):634-7.

51. Kalka C, Masuda H, Takahashi T, Kalka-Moll WM, Silver M, Kearney $\mathrm{M}$, et al. Transplantation of ex vivo expanded endothelial progenitor cells for therapeutic neovascularization. Proc Natl Acad Sci USA. 2000;97(7):3422-7.

52. Melero-Martin JM, Khan ZA, Picard A, Wu X, Paruchuri S, Bischoff J. In vivo vasculogenic potential of human blood-derived endothelial progenitor cells. Blood. 2007;109(11):4761-8.

53. Zhang X, Wei M, Zhu W, Han B. Combined transplantation of endothelial progenitor cells and mesenchymal stem cells into a rat model of isoproterenol-induced myocardial injury. Arch Cardiovasc Dis. 2008;101(5):333-42.

54. Leri A, Kajstura J, Anversa P. Cardiac stem cells and mechanisms of myocardial regeneration. Physiol Rev. 2005;85(4):1373-416.

55. Zimmet JM, Hare JM. Emerging role for bone marrow derived mesenchymal stem cells in myocardial regenerative therapy. Basic Res Cardiol. 2005;100(6):471-81.

56. Odeberg J, Piao JH, Samuelsson EB, Falci S, Akesson E. Low immunogenicity of in vitro-expanded human neural cells despite high MHC expression. J Neuroimmunol. 2005;161(1-2):1-11.

57. Cho PS, Messina DJ, Hirsh EL, et al. Immunogenicity of umbilical cord tissue_derived cells. Blood. 2008;111:430-438.

Avaliação: O tema apresentado consta da pauta elaborada pelo editor, Professor Milton Artur Ruiz, e coeditores deste suplemento, Professores Sergio Paulo Bydlowski e Adriana Seber.

Conflito de interesse: não declarado

Recebido: 03/10/2008

Aceito: $08 / 10 / 2008$ 